\title{
Ventriloquism in patients with unilateral visual neglect
}

\author{
Paul Bertelson ${ }^{\mathrm{a}, \mathrm{d}, *}$, Francesco Pavani ${ }^{\mathrm{b}, \mathrm{c}}$, Elisabetta Ladavas ${ }^{\mathrm{c}}$, Jean Vroomen ${ }^{\mathrm{d}}$, \\ Béatrice de Gelder ${ }^{\text {a, }}$ d \\ ${ }^{a}$ Laboratoire de Psychologie expérimentale, Université libre de Bruxelles, 50 Av. F. D. Roosevelt, B-1050, Bruxelles, Belgium \\ ' Ospedale INRCA "I Fraticini", Firenze, Italy \\ "Università di Bologna, Italy \\ d Tilburg University, The Netherlands
}

Received 30 June 1999; received in revised form 15 March 2000; accepted 30 March 2000

\begin{abstract}
Can visual stimuli that go undetected, because they are presented in the extinguished region of neglect patients' visual field, nevertheless shift in their direction the apparent location of simultaneous sounds (the well-known 'ventriloquist effect')? This issue was examined using a situation in which each trial involved the simultaneous presentation of a tone over loudspeakers, together with a bright square area on either the left, the right or both sides of fixation. Participants were required to report the presence of squares, and indicate by hand pointing the apparent location of the tone. Five patients with left hemineglect consistently failed to detect the left square, either presented alone or together with another square on the right. Nevertheless, on bimodal trials with a single undetected square to the left, their sound localization was significantly shifted in the direction of that undetected square. By contrast, in bimodal trials with either a single square on the right or a square on each side, their sound localization showed only small and non-significant shifts. This particular result might be due to a combination of low discrimination of lateral sound deviations with variable individual strategies triggered by conscious detection of the right square. The important finding is the crossmodal bias produced by the undetected left visual distractors. It provides a new example of implicit processing of inputs affected by unilateral visual neglect, and on the other hand is consistent with earlier demonstrations of the automaticity of crossmodal bias. (C) 2000 Elsevier Science Ltd. All rights reserved.
\end{abstract}

Keywords: Unilateral neglect; Ventriloquism; Implicit processing; Crossmodal interaction; Sound localization

\section{Introduction}

Studies of perceptual processing have generally been carried out within one particular input modality. But most real-life situations involve correlated sensory inputs to several modalities. The research that has dealt specifically with multimodal perception has identified many cases of cross-modal interaction, in which the interpretation of data in one modality is influenced by the information received in other modalities. This work has been based mainly on the imposition of experimental conflict between the data available to the involved modalities, as in the well-known case of prismatic displacement of the visual input [16].

\footnotetext{
* Corresponding author.

E-mail address: pbrtlsn@ulb.ac.be (P. Bertelson).
}

A type of conflict which has proved convenient for experimental study involves presentation of synchronous visual and auditory data in slightly separate locations. The processes put into play by such auditoryvisual (AV) conflict have been called ventriloquism because they are probably at the base of the illusion created by performing ventriloquists when the speech they produce without visible lip movements seems to originate from a simultaneously agitated puppet [3].

One of the main manifestations of ventriloquism is immediate cross-modal bias. It consists in the fact that when subjects are asked to indicate, by pointing or by some kind of verbal response, the location of inputs to one modality and to ignore spatially discordant ones in the other modality, the reported location of the target is displaced in the direction of the distractor $[2,6-$ $8,17,25,26,28,31]$. There is good evidence that a major condition for the occurrence of bias is synchronization 
of inputs to the two modalities [6,17,26,28,31]. Most studies of bias have been concerned with the visual bias of auditory localization, and the phenomenon has sometimes been attributed to 'visual capture', but evidence has been obtained for the existence of a smaller inverse effect, auditory bias of visual localization $[6,26]$.

One central question about spatial crossmodal bias concerns the locus of the underlying processes in the cognitive architecture. The usual demonstration of the phenomenon in the selective localization task consists in a partial failure to follow selective attention instructions. It might thus reflect the mandatory operation of a process which integrates spatial information across input modalities. However, that interpretation can only be accepted when the possible influence of some voluntary post-perceptual decisions has been excluded. The selective localization task is particularly susceptible to post-perceptual effects when the subject is aware of the intermodal spatial discrepancy (see discussion in [4]). However, in one earlier experiment, in which subjects presented with conflicting sound-light pairs were asked both to point to the sound and to judge the respective inputs as coming from a single source or two separate ones, significant visual bias of sound location was obtained not only on the trials on which the spatial discrepancy was detected, but also on those on which it was not [6]. More recent work, in which sound localization was measured through a staircase method, has confirmed that bias can occur under conditions precluding voluntary correction [5].

Useful information regarding the level of processing issue might be obtained by studying crossmodal interaction in patients with unilateral visual neglect. The neglect syndrome generally involves a reduced capacity to report visual stimuli in one visual hemifield, most often the one contralateral to the lesion $[11,13,29]$. One possible consequence of that condition could be that only visual stimuli presented to the intact field would bias auditory localization. An alternative possibility would be that even visual inputs directed to the neglected field, and which the patients do not detect explicitly, could nevertheless attract the apparent location of auditory targets. That kind of effect would of course be inconsistent with a post-perceptual interpretation of ventriloquism. Several studies with implicit measures of performance have shown that some forms of processing can be preserved in the affected field of patients with either visual neglect or visual extinction. For instance, such patients can to some extent compare stimuli across fields [1,30], their recognition of stimuli in the intact field can be facilitated by primes presented in the neglected field $[9,18]$, and their detection reaction time is faster with presentation in both hemifields than in the intact field alone [20].

The possibility that neglected visual stimuli could bias the apparent location of inputs to another modal- ity has to the best of our knowledge not been examined so far. One study with neglect patients has considered a form of crossmodal spatial bias [27], albeit one very different from the one used in the present investigation. The patients who recognized spoken syllables somewhat less accurately when delivered in their left auditory space than in their right auditory space, had this left side inferiority reduced when a dummy loudspeaker was visible in their right hemispace. Presumably, belief that the speech was coming from the dummy speaker moved its representation into the unaffected region of the patient's auditory space ${ }^{1}$. The important point to note about this result is that the dummy loudspeaker, which was located in the patients' spared space, was in all probability consciously perceived. Thus, the fact that its vision could bias the apparent direction of the speech by no means implies the sort of implicit processing of neglected data whose possibility was considered here.

In the present study, visual bias of auditory location was examined in patients with left hemifield neglect, using a situation in which sound bursts were accompanied by the simultaneous presentation on a monitor of spatially discordant visual distractors. Distractors were bright luminous squares appearing on the left, on the right or on both sides of the fixation point. Two main experimental tasks were administered. One was to describe the visual display, the other to point with the hidden hand to the apparent location of the sound. On each trial, the subjects also had to read aloud a small digit presented at fixation. This additional requirement played two roles: it imposed adequate gaze fixation, and, as will be explained in the method section, it probably contributed to have the patients miss the left distractor on a substantial proportion of trials.

Implicit processing of the undetected left distractor could affect the pattern of sound localization in two ways. First, the occurrence of a single undetected distractor on the left might shift sound localization in its direction. Second, on bilateral presentations, on which the patient would presumably report the right distrac-

\footnotetext{
${ }^{1}$ This kind of effect on auditory localization of seeing a sound producing object in a displaced location has been reported earlier for healthy subjects $([21,22]$, but see [25] for a failure to replicate). It is probably a completely different phenomenon from the one on which the present study is based. One difference concerns the timing of the inputs: the unchanging dummy loudspeaker continuously visible, and with no correlation with the changing speech signal as opposed, in the present task, to short bursts of sound and of light, with spatial interaction strongly dependent on synchronization. Another difference concerns processing level. The loudspeaker effect depends obviously on knowledge of the function of loudspeakers, a high level cognitive factor, while the AV bursts effect which requires no semantic association between the inputs nor any particular post-perceptual strategy, is automatic. As discussed elsewhere [3,4] a distinction should be made between an early, automatic, component of ventriloquism and later, more cognitive, components.
} 
Table 1

Personal and clinical data on the patients

\begin{tabular}{|c|c|c|c|c|c|c|c|c|c|}
\hline \multirow[t]{2}{*}{ Patient } & \multirow[t]{2}{*}{ Age } & \multirow[t]{2}{*}{ Sex } & \multirow[t]{2}{*}{ School years } & \multirow[t]{2}{*}{ Post-stroke months } & \multirow[t]{2}{*}{ Lesion $^{a}$} & \multicolumn{2}{|c|}{$\begin{array}{l}\text { Letter cancellation }{ }^{\mathrm{b}} \\
(\%)\end{array}$} & \multicolumn{2}{|c|}{$\begin{array}{l}\text { Bells cancellation }{ }^{c} \\
(\%)\end{array}$} \\
\hline & & & & & & $\mathrm{L}$ & $\mathrm{R}$ & $\mathrm{L}$ & $\mathrm{R}$ \\
\hline P1 & 68 & M & 5 & 4 & $\mathrm{~F}, \mathrm{~T}, \mathrm{P}$ & 25 & 86 & 0 & 39 \\
\hline $\mathrm{P} 2$ & 72 & M & 12 & 12 & $\mathrm{~F}, \mathrm{~T}, \mathrm{P}$ & 83 & 98 & 35 & 72 \\
\hline P34 & 69 & $\mathrm{~F}$ & 5 & 5 & $\mathrm{~T}, \mathrm{P}, \mathrm{sc}$ & 13 & 27 & 29 & 44 \\
\hline P6 & 66 & M & 5 & 4 & $\mathrm{~T}, \mathrm{P}$ & 87 & 100 & 82 & 94 \\
\hline
\end{tabular}

${ }^{\mathrm{a}} \mathrm{F}=$ frontal; $\mathrm{P}=$ parietal; $\mathrm{T}=$ temporal, occipital; $\mathrm{bg}=$ basal ganglia; $\mathrm{sc}=$ subcortical .

${ }^{\mathrm{b}}$ Diller \& Weinberg, 1977 [12].

${ }^{\mathrm{c}}$ Gauthier et al., 1989 [14].

tor only, the rightward shift of sound localization would nevertheless be smaller than on trials with presentation of a single right distractor.

\section{Method}

\subsection{Participants}

Six patients with left hemifield neglect consequent on ischemic lesions limited to the right hemisphere were tested. All were undergoing revalidation at the "I Fraticini" I.N.R.C.A. hospital in Florence. The clinical diagnosis was based on classical confrontation and cancellation tests (Letters test, [12]; Bells test, [14]). Inclusion in the study was also based on performance in a Unimodal Visual Detection task (see description in Section 2.2, Experimental tasks), which provided a direct assessment of patients' capacity to detect the visual distractors used to study crossmodal bias. Biographical and clinical data of the selected patients are given in Table 1. Although every patient had a lower performance on the left than on the right side of the page in both cancellation tasks, the difference was for some of them quite small. However, in the visual detection task, as well as the bimodal task, they missed most of the distractors presented in their left hemifield (see Section 3). The data of one patient (P6) were discarded from the analysis because he apparently failed to follow the selective attention instructions of our critical bimodal task (see Section 3).

Six control subjects with no neurological impairment, but ages as far as possible comparable to the patients (range 55-64; mean $=59.7$ ), were also tested. They were recruited among visitors to the hospital. All patients and control subjects gave informed consent to participate in the study.

\subsection{Experimental tasks}

The experimental set up is represented schematically in Fig. 1. It consists of a computer monitor located behind a pointing device, and of two loudspeakers, one on either side of the monitor. The $27 \times 20 \mathrm{~cm}$ monitor rested on a stand, which brought it about to the participants' eye level. The loudspeakers (each $12 \times$ $18 \mathrm{~cm}$ ) were hidden behind two screens made of black fabric, which prevented their vision, but did not attenuate the intensity of the sound nor change its apparent spatial location. Measured from the participant's head, the separation of the center of each loudspeaker from the center of the monitor was $19^{\circ}$ of angle. The pointing device consisted of a bowed rod fixated to the table top, in front of the monitor, and under an elevated horizontal panel which shielded it from the participant's sight. A graduated scale behind the rod allowed the experimenter to estimate the location of the pointing finger to the nearest degree of angle.

Three tasks were administered using that setup.

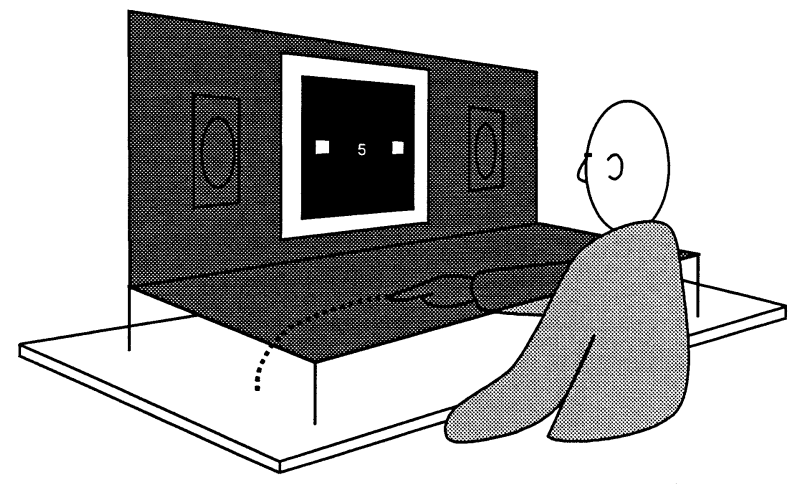

Fig. 1. Schematic view of the experimental setup for the bimodal task. 


\subsubsection{Unimodal visual detection task}

On each trial, a $5 \mathrm{~mm}$ high, $3 \mathrm{~mm}$ wide digit was presented for $120 \mathrm{~ms}$ in the center of the monitor screen in exact synchrony with either (a) a $20 \mathrm{~mm}$ $\left(1.9^{\circ}\right)$ white square with its axis $100 \mathrm{~mm}$ (or $9.5^{\circ}$ ) to the left (condition LS, for 'left square'), (b) $100 \mathrm{~mm}$ to the right (RS), (c) simultaneously on both sides of center $(2 \mathrm{~S})$ or (d) no square (NS). The location at which the digit occurred was marked by a white paper triangle pasted to the screen, and the participant was asked to keep his gaze fixated on that point. The instructions were to report the digit and then the rest of the visual display, by saying either 'square left', 'square right', 'two squares' or 'no square'. A total of 48 trials, 12 with each type of visual display, were administered in randomized order. The presentation of the visual display was accompanied with the synchronous delivery of a sound burst $(1000 \mathrm{~Hz}$ sine wave, $120 \mathrm{~ms}, 60 \mathrm{~dB}$ ) on the two loudspeakers, producing the impression of a single sound originating in a central location. In this task, the participant was not requested to do anything particular about that sound, which was only included to make the total sensory input the same as in the Bimodal Task.

The testing condition in which report of the central digit is requested on each trial was adopted after exploratory work showed that with the usual procedure in which the target of the monitoring task is presented only on a minority of catch trials (e.g. [6]), patients generally detected the left square on the no-digit trials but missed it when the digit was presented.

\subsubsection{Unimodal sound localization task}

A $120 \mathrm{~ms}, 60 \mathrm{~dB}$ burst of $1000 \mathrm{~Hz}$ sine wave sound was delivered on either the left, the right or simultaneously on both loudspeakers, and the participant was instructed to indicate the perceived location of the source by pointing with a finger of the right hand on the rod under the horizontal panel. The test consisted of 21 trials, seven for each location, in randomized order.

\subsubsection{Bimodal task}

This task combined the two preceding ones. On the 48 experimental trials of each session, the stimulation was the same as in the unimodal visual detection task: a central (bilateral) sound, a central visual digit and one of the four visual displays (LS, RS, $2 \mathrm{~S}$ or NS). Here, however, the participant had to point to the location of the sound before reporting on the digit and the squares. Eight trials on which the sound was delivered on either the left or the right loudspeaker only were interpolated randomly among the experimental trials, essentially to prevent the subjects from concluding that the sound always came from the same direction. The data from those trials were not included in the analysis.

Each patient participated in two sessions, on each of which the unimodal sound localization task and the bimodal task were administered, in that order. The unimodal visual detection task was administered only once, at the beginning of the first session, mainly to check that the patient failed to detect the left attractor on a sufficient proportion of trials, and also to familiarize him with reporting the central digit and the distractors, before adding, within the bimodal task, the requirement to point to the sound. Control subjects participated in one session only, during which they performed unimodal sound localization and the bimodal task. Pilot trials had shown that healthy subjects make practically no errors in the unimodal visual detection task.

\section{Results}

In the bimodal task, patients reported the central digit correctly on $83 \%$ of trials. However, many of the errors can be attributed to confusions between digits (such as reporting 1 for 7 , or 3 for 8 ) showing that on these trials fixation was close enough to extract substantial information from the target digit. This type or errors amount to $10 \%$, leaving a maximum of $7 \%$ of trials on which the required central fixation may not have been maintained.

The visual identification responses produced by the patients in the main bimodal task appear in Table 2 . These data are presented here in preference to those from the initial unimodal visual detection task, because they provide the information concerning detection of visual distractors for the very bimodal trials on which their sound biasing effect was measured. The right square was, with the exception of one RS trial, reported on every trial on which it was presented, whether alone (condition RS) or together with another square on the left (condition $2 \mathrm{~S}$ ). The left square went unreported on all but 14 of the $105 \mathrm{LS}$ trials, and on all but eight of the $1112 \mathrm{~S}$ trials, on which the right response was given. The few reports of the left square were nearly all produced by a single patient, P3, who gave 11 left responses on $21 \mathrm{LS}$ trials, and eight Bilateral responses on $222 \mathrm{~S}$ trials. Thus, except for the mixed results of that patient, the squares presented left of fixation were practically al- 
Table 2

Patients: Reports of visual distractors in the bimodal task

\begin{tabular}{llccc}
\hline & \multicolumn{2}{l}{ Visual display } & & \\
\cline { 2 - 5 } & No square (NS) & Left square (LS) & Two squares (2 S) & Right square (RS) \\
\hline $\begin{array}{l}\text { Nb. trials } \\
\text { Reports (\%) }\end{array}$ & 114 & 105 & 111 & 113 \\
$\quad$ None & 100.0 & 82.9 & - & - \\
$\quad$ Left & - & 13.3 & 7.3 & - \\
Bilateral & - & - & 92.7 & 99.1 \\
Right & - & 3.8 & & 99.1 \\
\hline
\end{tabular}

ways neglected, whether they were accompanied or not by a square in the right periphery ${ }^{2}$. Nevertheless, all trials with detection of the left distractor were discarded from the analysis of crossmodal bias.

Control subjects' visual identification in the bimodal task was error free.

To analyse the critical pointing data from the bimodal task, individual mean bias values were calculated by subtracting for each participant the mean pointing on the no-square trials from the mean pointing for each visual competition condition (LS, $2 \mathrm{~S}$, and RS). Trials on which a patient detected the presence of the left distractor were discarded from the analysis. Mean biases for each group appear in Fig. 2, together with $2 \mathrm{SE}$ (standard error) confidence intervals. Control subjects have comparable biases toward the left and the right square $\left(5.6^{\circ}\right.$ on the average), while their response in the bilateral condition shows no bias whatever. Patients, on the other hand, have much smaller biases toward the lateral squares.

Application of ANOVA to these data produced a non-significant main effect of group $\left(F_{1 \cdot 9}=2.48, p=\right.$ $0.15)$, a significant main effect of visual input $\left(F_{2: 18}=\right.$ 26.70; $p<0.001)$ and a significant group by visual input interaction $\left(F_{2 ; 18}=9.78 ; p=0.0013\right)$. This interaction probably results essentially from the reduction of lateral biases of the patients in comparison to those of the controls. The contrast between the groups is significant by $t$-test both for trials LS $\left(t_{10}=7.12 ; p<0.01\right)$ and RS $\left(t_{10}=4.03 ; p<0.02\right)$, but not for trials $2 \mathrm{~S}\left(t_{10}=1.15\right.$; $p=0.23)$.

\footnotetext{
${ }^{2}$ The question has been asked whether failures to report the left distractor really imply that it was not detected, rather than detected and then forgotten, while the patient was busy pointing to the sound. A partial answer to that question is provided by the detection performance of the patients in the initial unimodal visual detection task in which the memory load was smaller, since no pointing was imposed. In that task, reports of the left distractor were only marginally more frequent (15 reports for 58 LS and seven reports for 58 $2 \mathrm{~S}$ trials) than in the bimodal task (14/105 and 8/109). Thus, if detection followed by forgetting at all occurred, it was on a proportion of trials probably too small to affect the obtained bias.
}

The most important question to ask, however, is which of the biases displayed by each group in each of the three visual competition conditions (LS, RS and $2 \mathrm{~S}$ ) is significantly different from zero. For control subjects, the biases were significant by $t$-test in both conditions LS and RS (LS: $t_{5}=-4.44$, one-tailed $p=$ $0.004 ; \mathrm{RS}: t_{5}=5.83$, 1-tailed $p=0.002$ ). In condition $2 \mathrm{~S}$, mean bias was close to zero and in fact non-significant $\left(t_{5}=1.05, p=0.34\right)$. In patients, the bias was significant for condition LS $\left(t_{4}=-4.07\right.$; one-tailed $p=0.008)$, and non-significant for conditions RS $\left(t_{4}=\right.$ 1.62; one-tailed $p=0.09)$ and $2 \mathrm{~S}\left(t_{4}<1\right)$. The use of one-tailed tests is justified by the existence of clear a priori predictions regarding the direction of the effects. Note, however, that the leftward visual bias of the patients in condition LS would have been significant even with a two-tailed test.

A pattern of statistical significance similar to the one obtained with $t$-test is produced by application of the sign test: in condition LS, a leftward bias is observed in each of the five patients, which is significant $(5 / 5$, $p=0.031$ ), while in both conditions $\mathrm{RS}$ and $2 \mathrm{~S}$, a

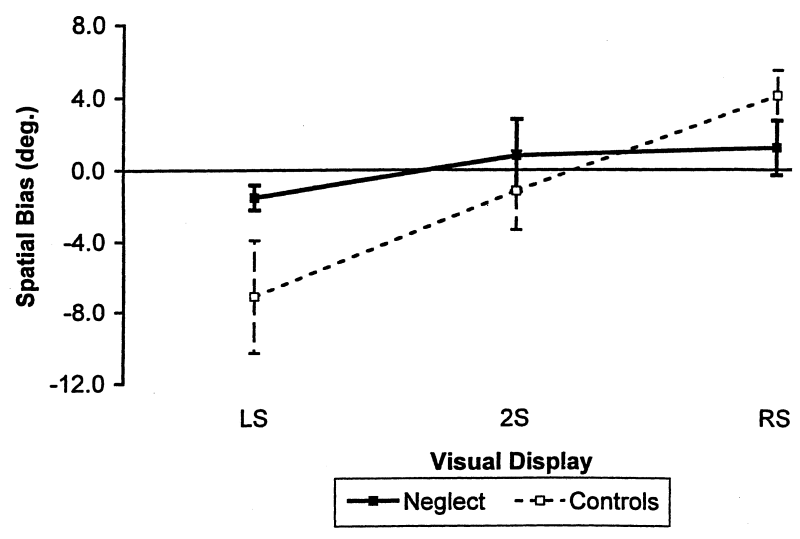

Fig. 2. Visual bias of sound localization in bimodal task: mean bias (in deg) per group (neglect patients, control subjects) and type of distractor (square to the left (LS), to the right (RS), or one square on each side $(2 \mathrm{~S})$. Bias is the difference between mean pointing in each condition with visual attractor(s) and in the no-square control condition $(\mathrm{NS})$. Positive bias values $=$ shift to the right; negative $=$ to the left. Brackets indicate 2 standard errors confidence intervals. 
rightward bias is observed in three patients only, which is non-significant $(3 / 5, p=0.50)$.

The immediate reason why the patients' rightward biases, unlike their leftward bias, fell short of significance is the fact, apparent in Fig. 2, that the inter-patients variability of the values is much larger in conditions $\mathrm{RS}\left(\mathrm{SD}=1.68^{\circ}\right)$ and $2 \mathrm{~S}\left(\mathrm{SD}=2.28^{\circ}\right)$ than in condition $\mathrm{LS}\left(\mathrm{SD}=0.83^{\circ}\right)$. A possible origin for that difference will be considered in Section 4 .

The patient (P6) whose data were discarded had the same pattern of visual detection performance as the other patients: he detected all occurrences of the right square and missed all those of the left square. However, his mean visual bias of sound localization was $-0.2^{\circ}$ (left) in condition LS and $+15.6^{\circ}$ and $+14.8^{\circ}$ (both right) in respectively conditions $\mathrm{RS}$ and $2 \mathrm{~S}$. The latter two bias values are larger than any obtained by a control subject, and also larger than the $9^{\circ}$ separation between the locations of the target sound and the right visual attractor. Actually, the patient pointed to the right of the right visual distractor on all but one of the trials under condition RS and on all trials under condition $2 \mathrm{~S}$. Maybe he misunderstood (or, for whatever reason, ignored) the instruction to base pointing on the sole auditory inputs, and instead pointed to the perceived location of the right square. That he overreached might be a manifestation of his condition ${ }^{3}$. The patient left the hospital soon after the experimental sessions, so that there was no opportunity to explore possible explanations for his strange performance through additional testing. But whatever the reason, his behavior was too different from that of the other patients to include him in a group analysis.

It is worth noting, on the other hand, that the pattern of statistical significance of the bias data from the group of patients would not have been different, had the results of P6 been included: $t$-tests would still be significant for LS $\left(t_{5}=3.46\right.$, one-tailed $p=0$. 009), and non-significant for $\mathrm{RS}\left(t_{5}=1.49, p=0.10\right)$ and $2 \mathrm{~S}$ $\left(t_{5}=1.25, p=0.14\right)$.

A possible explanation for the reduced visual biases exhibited by the patients was provided by their performance in the unimodal sound localization task. Mean pointing responses of the patients and of the control subjects in that task are shown in Fig. 3. Control subjects' responses to sounds from each of the lateral loudspeakers corresponded on the average fairly well with the actual locations of the sources (at $19^{\circ}$ left and right of center respectively), and their responses to sounds presented simultaneously on the two loudspeakers point, as expected, in the straight-ahead direction.

\footnotetext{
${ }^{3}$ It was suggested to us that the unusually large rightward shifts of P6 might reflect 'hyperattention'. Actually, there is now convincing evidence that the visual bias of auditory location is independent of where attention is directed [7].
}

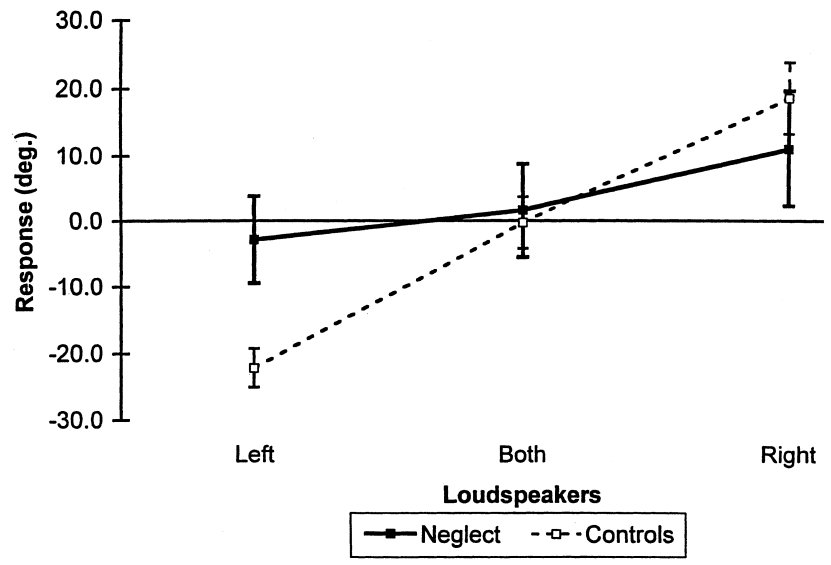

Fig. 3. Pointing in unimodal sound localization task: mean deviation (in deg) from straight ahead per group (neglect patients, control subjects) and sound origin (Left loudspeaker, Right loudspeaker, Both loudspeakers, i.e. simultaneously and at same loudness on both). Positive deviations $=$ to the right, negative $=$ to the left. Brackets indicate 2 standard errors confidence intervals.

In contrast, patients' mean responses to sounds from the lateral loudspeakers fell systematically short of their actual eccentricity, particularly for sounds presented in left space.

Responsiveness to eccentricity was measured by the distance between mean pointing for each lateral location (left or right loudspeaker) and that for the central location (both loudspeakers). That distance was computed for each subject and compared to the objective distance between central location and each lateral loudspeaker, $19^{\circ}$. For patients, pointing fell significantly short of the predicted $19^{\circ}$ (left loudspeaker: by $14.6^{\circ}$, $t_{4}=9.95, p<0.001$; right loudspeaker: by $9.7^{\circ}, t_{4}=$ 9.97, $p<0.001)$. Although the underestimation was significantly stronger in the left hemispace than in the right one $\left(t_{4}=3.62, p=0.011\right)$, the important point is that responsiveness to eccentricity was reduced significantly in both hemispaces. This reduction might be the reason why their visual biases, even the significant leftward biases from the left distractor, were so much smaller than those exhibited by control subjects. This point will be considered again in Section 4.

In Fig. 3, the patients' mean pointing to central (i.e. both loudspeakers) auditory stimulation is, like that of the controls, close to straight ahead. Individual data produce however a more complex picture: four patients (P1, P3, P4 and P5) show a rightward mean deviation (respectively $1.1,9.6,2.9$, and $5.7^{\circ}$ ) which is masked at the level of the group's mean by a strong leftward deviation $\left(-11.4^{\circ}\right)$ displayed by the fifth patient, P2. There is no completely convincing explanation for this particular behavior. However, P2's sound localization showed, like that of the other patients, some limited influence of source location (contrast between mean pointings to sounds from respectively left and both loudspeakers: $-3.8^{\circ}$; between right and both: $+7.3^{\circ}$ ). This suggests that this patient's leftward pointing ten- 
dency might be of post-perceptual origin. Regarding the other measures of performance, P2 had total left visual extinction and his visual biases (LS: $-1.0^{\circ}$, RS: $+2.5^{\circ}, 2 \mathrm{~S}:-1.5^{\circ}$ ) were within the range of those of the four other patients, P1, P3, P4 and $\mathrm{P} 5^{4}$.

\section{Discussion}

The primary purpose of our study was to examine the possibility of a new case of implicit processing of not explicitly detected inputs. Specifically, we asked if a well-known phenomenon, the attraction of the apparent location of a sound toward a simultaneously occurring but spatially discordant visual distractor (the ventriloquist effect), also takes place when the distractor goes for some reason undetected. Patients with left visual neglect provided the opportunity for an answer. In the task we used, with on each trial central presentation of a digit that they had to report, they displayed near total neglect of distractors presented in their impaired left hemifield.

To our knowledge, this particular task has not been used before in work on neglect. It was adopted after exploratory trials, in which patients monitored the central area for the occasional occurrence of a to be reported digit, showed that they only neglected left distractors when a digit was actually presented. This difference in the determination of neglect between mere monitoring and deeper processing of a central target may have important implications concerning the mechanism of neglect and particularly its relation to the allied symptom of extinction [13,29]. The question was not pursued within the present study, but it is the focus of a program carried out currently in Tilburg.

In the healthy control subjects, the usual attraction of perceived sound location toward a synchronous visual distractor was obtained with both unilateral distractors, whether left or right. On the other hand, no bias was observed with bilateral distractors, which confirms an earlier finding that, under that condition, the attractions exerted by the two distractors effectively cancel each other [6]. Thus, habitual crossmodal biases were obtained in an experimental situation different from several points of view from those used in earlier studies (single sound-light pulses instead of trains of

\footnotetext{
${ }^{4}$ The case of P2 is very different from that of P6, whose data we decided not to analyze together with those of the rest of the group. P2's singularity occurred in a control task, for which no prediction existed based on either established theory or results in the literature. On the other hand, when his sound localization performance in the critical bimodal task were analyzed, like for the other patients, as deviations from his own baseline on control NS trials with no visual distractor, it was comparable to that of the other patients. There was thus no reason to separate the data of $\mathrm{P} 2$ from those of the remaining patients, as was done for P6.
}

several pulses; visual distractors presented on a computer monitor instead of LED lamps; report of central digit on every trial), and with subjects older and less educated than the usual university undergraduates.

The important new finding is the leftward shift in sound localization observed in neglect patients when a single visual distractor was presented in left space (condition LS) and went undetected. The bias was much smaller than in control subjects, but nevertheless significant.

On the other hand, in the conditions with a single visual attractor to the right (condition RS) or visual attractors on both sides (condition $2 \mathrm{~S}$ ), the effects of the visual inputs were not only small but also highly variable. Consequently, they fell short of significance for condition RS and far below for condition $2 \mathrm{~S}$.

Patients' reduced biases may be a reflection of the reduced responsiveness of their auditory pointing to the degree of target eccentricity, which was revealed by their performance in the unimodal sound localization task. It was apparently the case that reduced responsiveness applies to eccentricities produced by the crossmodal bias just as to those produced by actual lateral displacement of the auditory source.

The fact which remains to be explained is why interpatient variability was bigger for rightward than for leftward bias, which is apparently the reason why the former failed to reach statistical significance. One possible explanation can be found in the notion that biases measured in normal subjects comprise an automatic shift toward the attractor plus possibly some voluntary postperceptual adjustments, variable within and between subjects $[4,26]$. Several results in the literature are consistent with this two-factor view. One is the fact that when immediate visual bias of sound location was measured on the same subjects as aftereffects, it was found to have much larger variability [25]. Auditory aftereffects are measured in a unimodal localization task, which presumably leaves less room for postperceptual adjustments than the selective localization task in which crossmodal bias is usually studied. The extent to which deliberate strategies can vary is further illustrated by the fact that a significant negative bias, i.e. a shift of auditory pointing away from the visual attractor, has been obtained in one study at least [24]. That result presumably reflected a voluntary effort on the part of the subjects to resist the visual attraction.

The explanation we are proposing for the pattern of visual bias observed in our patients is thus that their leftward bias reflects the sole operation of the automatic component, but that the consciously perceived right attractor triggered both the automatic and the voluntary component. The absence of significant rightward bias would be the combined result of low sensitivity to eccentricity and of the variability of voluntary strategies. 
How typical of visual neglect is the pattern of sound localization displayed by our patients is difficult to say, because of the paucity of relevant data in the literature. A tendency to displace to the right the apparent location of sounds from a median source has been described in several studies with neglect patients $[10,19,23]$. It was replicated by four of our five patients. The leftward displacement observed in the fifth one (P2) was probably, we argued, of postperceptual origin. Another aspect of the performance of our patients, reduced responsiveness to sound eccentricity in both half spaces, has to our knowledge been reported in only one other study [15], in which it was present also in healthy controls. Reduced responsiveness to sound eccentricity limited to the left hemispace was observed in two of the already mentioned studies, one with a single case [19] and the other with three cases, two of whom presented the pattern [23]. In the third study [10], which concerned the apparent location within patients' heads of stimuli presented stereophonically over earphones, responsiveness to right side eccentricity could not be assessed, due to a ceiling effect. Further work with patients is clearly needed to determine the conditions under which unilaterally or bilaterally reduced responsiveness to eccentricity occurs.

Let us now return to the main question whether neglected visual stimuli can bias the perceived location of sounds. In the Introduction, two possible effects of the attraction of target sounds by a neglected left square were predicted: a leftward bias on trials with a single left distractor (condition LS), and on trials with bilateral distractors (condition $2 \mathrm{~S}$ ) a rightward bias smaller than on those with a single right distractor (condition RS).

The first prediction was supported by the fact that our patients displayed a small but significant bias toward the undetected single left distractor in condition LS. We have proposed that the small size of that effect can be explained by the reduced responsiveness of the patients' pointing behavior to the eccentricity of auditory targets.

The second prediction could not be tested because, contrary to our expectation, the patients showed no significant bias toward the single right distractor in condition RS. This state of affairs, we argued, could have resulted from a combination of low responsiveness to eccentricity and variable strategies in dealing with the consciously detected right distractor. Thus, the prediction was not actually rejected, the conditions for testing it were simply not present in this study.

In conclusion, evidence of attraction of sounds apparent location in the direction of undetected visual stimuli has been obtained. That result provides a new example of behavioral effectiveness of stimuli which went undetected because they fell in the neglected region of patients' visual field. Earlier examples of such implicit processing have been concerned either with identification $[1,9,18,30]$ or detection speed [20] and the present one would be the first dealing with localization performance.

Concerning the mechanism of ventriloquism, the finding that visual bias can be obtained in the absence of awareness of the occurrence of the visual attractor provides a strong argument for the automaticity of the phenomenon. The argument is stronger than earlier ones based on the fact that bias could be obtained with healthy subjects in situations in which they were not aware of the spatial discrepancy between auditory target and visual attractor, but were of course aware of the occurrence of both auditory target and visual attractor $[5,6]$.

\section{Acknowledgements}

The present work was supported by the Ministry of Scientific Research of the Belgian French-speaking Community (Concerted Research Actions 91/96-148 and 96/01-2037) and by the Belgian National Fund for Collective Fundamental Research. The assistance of the Technical department of Tilburg University for setting up the testing situation is gratefully acknowledged. Thanks are due to three anonymous reviewers for constructive criticisms and to Carlo Umiltà for many insightful comments on previous versions of the paper.

\section{References}

[1] Baylis GC, Driver J, Rafal RD. Visual extinction and stimulus repetition. Journal of Cognitive Neuroscience 1993;5:453-66.

[2] Bermant RI, Welch RB. The effect of degree of visual-auditory stimulus separation and eye position upon the spatial interaction of vision and audition. Perceptual and Motor Skill 1976;43:48793.

[3] Bertelson P. Starting from the ventriloquist: The perception of multimodal events. In: Sabourin M, Craik FIM, Robert M, editors. Advances in psychological science, Vol. 2: biological and cognitive aspects. Hove, UK: Psychology Press, 1988:419-39.

[4] Bertelson P. Ventriloquism: a case of crossmodal perceptual grouping. In: Aschersleben G, Bachmann T, Müsseler J, editors. Cognitive contributions to the perception of spatial and temporal events. Amsterdam: Elsevier, 1999:347-62.

[5] Bertelson P, Aschersleben G. Automatic visual bias of auditory location. Psychonomic Bulletin \& Review 1998;5:482-9.

[6] Bertelson P, Radeau M. Cross-modal bias and perceptual fusion with auditory-visual spatial discordance. Perception and Psychophysics 1981;29:578-87.

[7] Bertelson P, Vroomen J, de Gelder B, Driver J. The ventriloquist effect does not depend on the direction of deliberate visual attention. Perception \& Psychophysics 2000;62:321-32

[8] Bertelson P, Vroomen J, Wiegeraad G, de Gelder B. Exploring the relation between McGurk interference and ventriloquism. In: International Congress on Spoken language Processing (ISCLP), Yokohama, 1994:559-62.

[9] Berti A, Rizzolatti G. Visual processing without awareness: evidence from unilateral neglect. Journal of Cognitive Neuroscience 1992;4:345-51. 
[10] Bisiach E, Cornacchia L, Sterzi R, Vallar G. Disorders of perceived auditory lateralization after lesions of the right hemisphere. Brain 1984;107:37-52.

[11] Bisiach E, Vallar G. Hemineglect in humans. In: Boller F, Grafman J, editors. Handbook of neuropsychology, vol. vol. I. Elsevier: Amsterdam, 1988:195-222.

[12] Diller L, Weinberg J. Hemi-inattention in rehabilitation: the evolution of a rational remediation program. In: Weinstein EA, Friedland RP, editors. Hemi-inattention and hemispheric specialization. Advances in neurology. New York: Raven Press, 1977.

[13] Driver J, Mattingley JB, Rorden C, Davis G. Extinction as a paradigm measure of attentional bias and restricted capacity following brain injury. In: Their P, Karnath H-O, editors. Parietal lobe contributions to orientation in 3-D space. Springer, 1997:401-29.

[14] Gauthier L, Dehaut F, Joannette Y. The bells test: a quantitative and qualitative test for visual neglect. International Journal of Clinical Neuropsycology 1989;11:49-54.

[15] Haeske-Dewick H, Canavan AGM, Homberg V. Sound localization in egocentric space following hemispheric lesions. Neuropsychologia 1996;34:937-42.

[16] Held R. Plasticity in sensorimotor systems. Scientific American 1965;213:84-94.

[17] Klemm O. Localization von Sinneneindrücken bei disparate Nebenreizen. Psychologische Studien (Wundt) 1909;5:73-161.

[18] Ladavas E, Paladini R, Cubelli R. Implicit associative priming in a patient with left visual neglect. Neuropsychologia 1993;31:130720.

[19] Ladavas E, Pavani F. Neuropsychological evidence of the functional integration of visual, auditory and proprioceptive spatial maps. NeuroReport 1999;9:1195-200.

[20] Marzi CA, Smania N, Martini MC, Gambina G, Tomelleri G, Palamara A, Alessandrini F, Prior M. Implicit redundant-targets effect in visual extinction. Neuropsychologia 1996;34:9-22.
[21] Morais J. The effect of ventriloquism on the right-side advantage for verbal material. Cognition 1974-75;3:127-39.

[22] Pick HL, Warren DH, Hay JC. Sensory conflict in judgements of spatial direction. Perception \& Psychophysics 1969;6:203-5.

[23] Pinek B, Duhamel J-R, Cavé C, Brouchon M. Audio-spatial deficit in humans: differential effects associated with left versus right hemisphere parietal damage. Cortex 1989;25:175-86.

[24] Radeau M. Adaptation au déplacement prismatique sur la base d'une discordance entre la vision et l'audition. L'Année Psychologique 1974; 74:23-34.

[25] Radeau M. Cognitive impenetrability in auditory-visual interaction. In: Alegria J, Holender D, Morais J, Radeau M, editors. Analytic approaches to human cognition. Amsterdam: Elsevier, 1992:41-55.

[26] Radeau, Bertelson. Auditory-visual interaction and the timing of inputs: Thomas (1941) revisited. Psychological Research 1987;49:17-22.

[27] Soroker N, Calamaro N, Myslobodsky MS. Ventriloquism effect reinstates responsiveness to auditory stimuli in the ignored' space in patients with hemispatial neglect. Journal of Clinical and Experimental Neuropsychology 1995;17:243-55.

[28] Thomas GJ. Experimental study of the influence of vision on sound localization. Journal of Experimental Psychology 1941;28:167-77.

[29] Vallar G. Spatial hemineglect in humans. Trends in Cognitive Sciences 1998;2:87-97.

[30] Volpe BT, Ledoux J, Gazzaniga MS. Information processing of visual stimuli in an "extinguished" field. Nature 1979;282:722-4.

[31] Warren DH, Welch RB, McCarthy TJ. The role of visual-auditory "compellingness" in the ventriloquism effect: implications for transitivity among the spatial senses. Perception \& Psychophysics 1981;30:557-64. 